\title{
Crystal structure of bis(2-(2-((2,6-dichlorophenyl) amino)phenyl)acetato- $\left.\mathrm{K}^{2} O, 0^{\prime}\right)-(1,10-$ phenanthroline- $\left.\mathrm{K}^{2} \mathrm{~N}, \mathrm{~N}^{\prime}\right)$ zinc(II), $\mathrm{C}_{40} \mathrm{H}_{28} \mathrm{Cl}_{4} \mathrm{~N}_{4} \mathrm{O}_{4} \mathrm{Zn}$
}

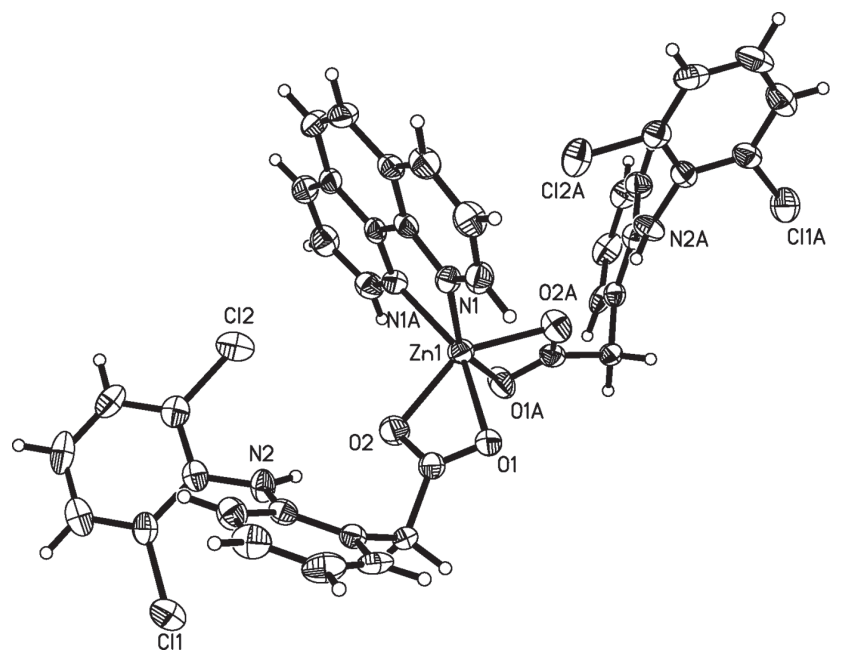

https://doi.org/10.1515/ncrs-2020-0212

Received April 30, 2020; accepted June 10, 2020; available online June 26,2020

\section{Abstract}

$\mathrm{C}_{40} \mathrm{H}_{28} \mathrm{Cl}_{4} \mathrm{~N}_{4} \mathrm{O}_{4} \mathrm{Zn}$, monoclinic, $\mathrm{C2} / c$ (no. 19), $a=26.573(4) \AA$, $b=12.898(2) \AA, \quad c=11.4503(17) \AA, \quad \beta=110.837(12)^{\circ}$, $V=3667.8(10) \AA^{3}, Z=4, R_{\mathrm{gt}}(F)=0.0631, w R_{\mathrm{ref}}\left(F^{2}\right)=0.1100$, $T=293(2) \mathrm{K}$.

\section{CCDC no.: 2009085}

The molecular structure of the title complex is shown in the figure. Table 1 contains crystallographic data and Table 2 contains the list of the atoms including atomic coordinates and displacement parameters.

\section{Source of material}

A mixture of $\mathrm{Zn}(\mathrm{OAc})_{2} \cdot 2 \mathrm{H}_{2} \mathrm{O} \quad(22 \mathrm{mg}, \quad 0.1 \mathrm{mmol})$, 1,10-phenanthroline $(0.1 \mathrm{mmol}, 18 \mathrm{mg})$, and 2-(2-(2,6dichlorophenylamino)phenyl)acetic acid (H2dpa) (0.1 mmol,

\footnotetext{
*Corresponding author: Yan Zhao, Physics and Electronic Information College, Luoyang Normal University, Luoyang, Henan 471934, P.R. China, e-mail: luoyangchangchun@126.com. https://orcid.org/0000-0002-1723-7683

Shi-Hui Li: College of Chemistry and Chemical Engineering, Luoyang Normal University, Luoyang, Henan 471934, P.R. China
}

Table 1: Data collection and handling.

\begin{tabular}{ll}
\hline Crystal: & Colourless block \\
Size: & $0.42 \times 0.38 \times 0.26 \mathrm{~mm}$ \\
Wavelength: & Mo $K \alpha$ radiation $(0.71073 \AA$ A $)$ \\
$\mu:$ & $1.01 \mathrm{~mm}^{-1}$ \\
Diffractometer, scan mode: & SuperNova, $\omega$ \\
$\theta_{\text {max }}$, completeness: & $25.5^{\circ}, 98 \%$ \\
$N\left(h k l_{\text {measured }}, N(h k l)_{\text {unique }}, R_{\text {int }}:\right.$ & $7005,3329,0.048$ \\
Criterion for $I_{\text {obs }}, N(h k l)_{\mathrm{gt}}:$ & $I_{\text {obs }}>2 \sigma\left(I_{\text {obs }}\right), 1966$ \\
$N(\text { param })_{\text {refined }}:$ & 240 \\
Programs: & CrysAlis ${ }^{\text {PRO }}[1]$, SHELX $[2,3]$ \\
\hline
\end{tabular}

Table 2: Fractional atomic coordinates and isotropic or equivalent isotropic displacement parameters $\left(\AA^{2}\right)$.

\begin{tabular}{lrrrr}
\hline Atom & $\boldsymbol{x}$ & $\boldsymbol{y}$ & $\boldsymbol{z}$ & $\boldsymbol{U}_{\text {iso }}{ }^{*} \boldsymbol{U}_{\text {eq }}$ \\
\hline Zn1 & 1.0000 & $0.26895(6)$ & 1.2500 & $0.0470(3)$ \\
Cl1 & $0.73219(5)$ & $0.35914(12)$ & $0.71525(12)$ & $0.0754(5)$ \\
Cl2 & $0.84789(5)$ & $0.09311(11)$ & $1.07578(11)$ & $0.0707(4)$ \\
O1 & $1.00073(13)$ & $0.3776(3)$ & $1.0914(3)$ & $0.0767(12)$ \\
O2 & $0.92965(12)$ & $0.3169(3)$ & $1.1175(3)$ & $0.0626(10)$ \\
N1 & $1.02997(13)$ & $0.1428(3)$ & $1.1774(3)$ & $0.0425(9)$ \\
N2 & $0.83447(13)$ & $0.2720(3)$ & $0.9023(3)$ & $0.0497(10)$ \\
H2 & 0.8519 & 0.2944 & 0.9764 & $0.060^{*}$ \\
C1 & $0.9515(2)$ & $0.3733(4)$ & $1.0582(4)$ & $0.0496(13)$ \\
C2 & $0.91497(15)$ & $0.4339(3)$ & $0.9460(3)$ & $0.0427(12)$ \\
H2A & 0.8842 & 0.4592 & 0.9641 & $0.051^{*}$ \\
H2B & 0.9345 & 0.4935 & 0.9325 & $0.051^{*}$ \\
C3 & $0.89525(16)$ & $0.3699(3)$ & $0.8277(4)$ & $0.0403(11)$ \\
C4 & $0.91605(18)$ & $0.3899(4)$ & $0.7346(4)$ & $0.0518(13)$ \\
H4 & 0.9432 & 0.4390 & 0.7483 & $0.062^{*}$ \\
C5 & $0.8968(2)$ & $0.3374(4)$ & $0.6209(5)$ & $0.0674(16)$ \\
H5 & 0.9102 & 0.3529 & 0.5581 & $0.081^{*}$ \\
C6 & $0.8583(2)$ & $0.2632(4)$ & $0.6027(4)$ & $0.0621(15)$ \\
H6 & 0.8458 & 0.2270 & 0.5276 & $0.075^{*}$ \\
C7 & $0.83759(17)$ & $0.2411(4)$ & $0.6955(4)$ & $0.0553(14)$ \\
H7 & 0.8114 & 0.1902 & 0.6822 & $0.066^{*}$ \\
C8 & $0.85598(17)$ & $0.2950(4)$ & $0.8087(4)$ & $0.0428(12)$ \\
C9 & $0.78783(18)$ & $0.2164(4)$ & $0.8849(4)$ & $0.0468(13)$ \\
C10 & $0.73801(19)$ & $0.2442(4)$ & $0.7985(4)$ & $0.0532(13)$ \\
C11 & $0.6925(2)$ & $0.1869(5)$ & $0.7809(5)$ & $0.0710(17)$ \\
H11 & 0.6600 & 0.2066 & 0.7203 & $0.085^{*}$ \\
C12 & $0.6947(2)$ & $0.1003(5)$ & $0.8526(6)$ & $0.0746(18)$ \\
H12 & 0.6639 & 0.0609 & 0.8395 & $0.090^{*}$ \\
C13 & $0.7422(2)$ & $0.0721(4)$ & $0.9431(5)$ & $0.0628(14)$ \\
& & & &
\end{tabular}


Table 2 (continued)

\begin{tabular}{lrrrr}
\hline Atom & $\boldsymbol{x}$ & $\boldsymbol{y}$ & $\boldsymbol{z}$ & $\boldsymbol{U}_{\text {iso }}{ }^{*} \boldsymbol{U}_{\text {eq }}$ \\
\hline H13 & 0.7435 & 0.0152 & 0.9940 & $0.075^{*}$ \\
C14 & $0.78830(18)$ & $0.1286(4)$ & $0.9586(4)$ & $0.0511(13)$ \\
C15 & $1.01730(15)$ & $0.0496(4)$ & $1.2141(4)$ & $0.0404(11)$ \\
C16 & $1.03460(16)$ & $-0.0444(4)$ & $1.1796(4)$ & $0.0449(12)$ \\
C17 & $1.06833(17)$ & $-0.0396(4)$ & $1.1096(4)$ & $0.0569(14)$ \\
H17 & 1.0817 & -0.1001 & 1.0874 & $0.068^{*}$ \\
C18 & $1.08143(18)$ & $0.0544(5)$ & $1.0742(4)$ & $0.0634(15)$ \\
H18 & 1.1036 & 0.0587 & 1.0271 & $0.076^{*}$ \\
C19 & $1.06111(17)$ & $0.1446(4)$ & $1.1095(4)$ & $0.0552(14)$ \\
H19 & 1.0699 & 0.2084 & 1.0842 & $0.066^{*}$ \\
C20 & $1.01663(17)$ & $-0.1390(4)$ & $1.2170(4)$ & $0.0570(14)$ \\
H20 & 1.0281 & -0.2019 & 1.1954 & $0.068^{*}$ \\
\hline
\end{tabular}

$891 \mathrm{mg}$ ), was dissolved in $9 \mathrm{~mL} \mathrm{H}_{2} \mathrm{O}$. The solution was heated in a $23 \mathrm{~mL}$ Teflon-lined autoclave under autogenous pressure at $373 \mathrm{~K}$ for 4 days. After cooling to room temperature colorless crystals formed.

\section{Experimental details}

Coordinates of hydrogen atoms were added using various restraints. Their $U_{\text {iso }}$ values were set to $1.2 U_{\text {eq }}$ of the parent atoms.

\section{Comment}

The design and construction of MOFs is based on organic ligands, which are different in size, exibility, coordination ability and so on [4]. In most situations, $N$-containing ligands are electrically neutral, while carboxylate ligands are negatively charged in these compounds. Among organic aromatic carboxylates, the 2-(2-(2,6-dichlorophenylamino)phenyl)acetate can yield predetermined networks [5-8]. On the other hand, 1,10-phenanthroline serves as co-ligand [9-11].

In the title complex, there is one half of a $\mathrm{Zn}(\mathrm{II})$, one Hdpa, and one half of the 1,10-phenanthroline ligand in the asymmetric unit (cf. the figure). $\mathrm{Zn} 1$ is six-coordinated with a distorted octahedral geometry by four $\mathrm{O}$ atoms from the Hdpa ligands, and two $\mathrm{N}$ atoms from one 1,10-phenanthroline ligand. The $\mathrm{Zn}-\mathrm{N}$ bond length is 2.108(4) $\AA$; and the $\mathrm{Zn}-\mathrm{O}$ ones are in the range of 2.041(3) to 2.298(4) $\AA$, respectively. The bond angles of $\mathrm{O}-\mathrm{Zn}-\mathrm{O}$ are in the range of $59.36(12)^{\circ}$ to $144.6(2)^{\circ}$. All geometric parameters are comparable to related ones $[12,13]$. There is a hydrogen bond between each 02 oxygen atom and the $\mathrm{NH}$ group of the organic ligands: $d(\mathrm{~N} \cdots \mathrm{O})$ : 2.893(5) $\AA$ and $\mathrm{N}-\mathrm{H}$. . O O is $145.7 \AA$.
Acknowledgements: The authors thank the Key Scientific Research Project for Universities of Henan Province (No. 18B150015; No. 20A150028) and Science and Technology Research Project of Henan Province (no. 182102310909) for financial support.

\section{References}

1. Agilent Technologies: CrysAlis ${ }^{\mathrm{PRO}}$ Software system, version 1.171.35.15. Agilent Technologies UK Ltd, Oxford, UK (2011).

2. Sheldrick, G. M.: SHELXT - Integrated space-group and crystalstructure determination. Acta Crystallogr. A71 (2015) 3-8.

3. Sheldrick, G. M.: Crystal structure refinement with SHELXL. Acta Crystallogr. C71 (2015) 3-8.

4. Li, S. H.; Zang, J.; Guo, J. B.: Synthesis and crystal structure of a new three-dimensional Ho(II) coordination polymer based on 5'-(4-carboxyphenyl)-[1,1': $3^{\prime}, 1^{\prime \prime}$-terphenyl]-4,4'-dicarboxylic acid. Inorg. Nano-Met. Chem. 12 (2017) 1746-1749.

5. Guo, J. B.; Li, S. H.: Crystal structure of bis(4-(1H-pyrazol5-yl)pyridine- $\left.{ }^{2} N: N^{\prime}\right)$-bis(2-(2-((2,6-difluorophenyl)amino) phenyl)acetate- $\mathrm{k} O$ )cadmium(II). Z. Kristallogr. NCS 232 (2017) 969-970.

6. Duan, Y.; Li, S. H.: Crystal structure of catena-poly[diaqua-bis ( $\mu_{2}$-2-(2-((2,6-dichlorophenyl)amino)phenyl)acetato- $\left.\mathrm{K}^{3} \mathrm{O}, \mathrm{O}^{\prime}: \mathrm{O}^{\prime}\right)$ calcium(II)]. Z. Kristallogr. NCS 233 (2018) 169-171.

7. Li, S. H.; Zhao, Y.; Zhang, L.: Crystal structure of catena-poly[( $\mu_{2}-2-\left(2-\left(\left(2,6-\right.\right.\right.$ dimethylphenyl)amino)phenyl)acetato- $\left.\kappa^{2} O: O^{\prime}\right)$ ( $\mu_{2}$-2-(2-((2,6-dimethylphenyl)amino)phenyl) acetate- $\kappa^{3} O, O^{\prime}$ : $\left.O^{\prime}\right)$ cadmium(II)]. Z. Kristallogr. NCS 231 (2016) 1009-1011.

8. Song, W.; Xu, X. L.; Yang, Y. J.; Li, S. H.: Synthesis and crystal structure of catena-poly[bis(2-(2-((2,6-dichlorophenyl) amino)phenyl) acetato- $\left.\mathrm{k} O ; \mathrm{K}^{2} \mathrm{O}^{\prime}, \mathrm{O}^{\prime \prime}\right)-\left(\mu_{2}-1,4\right.$-di(pyridin-4yl)benzene- $\left.\kappa^{2} N: N^{\prime}\right)$ zinc(II)]. Z. Kristallogr. NCS 233 (2018) 399-401.

9. Song, W.; Xu, X. L.; Yang, Y. J.; Li, S. H.: Crystal structure of catena-poly $\left[\left(\mu_{3}-5\right.\right.$-carboxy-2-(pyridin-4-yl)benzoato- $\mathrm{K}^{5} \mathrm{O}$, $\left.O^{\prime}: O^{\prime \prime}, O^{\prime \prime \prime}: O^{\prime \prime \prime}\right)\left(1,10\right.$-phenanthroline- $\left.{ }^{2} N, N^{\prime}\right)$ cadmium(II)]. Z. Kristallogr. NCS 233 (2018) 755-756.

10. Chen, S. J.; Li, S. H.: Crystal structure of tetra-aqua-bis(2thiophene-3,4-dicarboxylato- $\left.{ }^{4} O, O^{\prime}: O^{\prime \prime}, O^{\prime \prime \prime}\right)$-bis (thiophene3-carboxyl-4-carboxylato- $\mathrm{K}^{1} O$ )-bis(1,10-phenantroline- $\left.\mathrm{K}^{2} N, N^{\prime}\right)$ disamarium(III). Z. Kristallogr. NCS 230 (2015) 259-260.

11. Guo, C. Y.; Li, S. H.: Synthesis and crystal structure of a $\mathrm{Ni}(\mathrm{II})$ complex with 4-bromoisophthalic acid and 1,10phenanthroline. Synth. React. Inorg. Met.-Org. Nano-Met. Chem. 42 (2012) 902-904.

12. Abu, A. H.; Jabali, B.: Synthesis, characterization and biological activity of novel complexes of zinc(II) diclofenac with nitrogen based ligands. Polyhedron 107 (2016) 97-106.

13. Perontsis, S.; Dimitriou, A.; Fotiadou, P.; Hatzidimitriou, A. G.; Papadopoulos, A. N.; Psomas, G.: Cobalt(II) complexes with the non-steroidal anti-inflammatory drug diclofenac and nitrogen-donor ligands. J. Inorg. Biochem. 196 (2019) 110688. 